J3eA, Journal sur l'enseignement des sciences et technologies de l'information et des systèmes, Volume 2, Hors-Série 2, 17 (2003)

DOI : http://dx.doi.org/10.1051/bib-j3ea:2003617

(C) EDP Sciences, 2003

Endoscope à béquillage actif : intégration d'actionneurs/capteurs

J. Szewczyk, V. de Sars et P. Bidaud

Laboratoire de Robotique de Paris 6

18 , route du panorama

BP 61

F-92265 Fontenay aux Roses Cedex, France

szewczyk@robot. jussieu. fr 


\section{Endoscope à béquillage actif : intégration d'actionneurs/capteurs.}

\author{
J. Szewczyk \\ Laboratoire de Robotique de Paris 6 \\ 18 , route du panorama BP 61 \\ 92265 Fontenay aux Roses Cedex -France
}

\author{
V. de Sars, Ph. Bidaud \\ Laboratoire de Robotique de Paris 6 \\ 18 , route du panorama BP 61 \\ 92265 Fontenay aux Roses Cedex -France
}

E-mail :.szewczykerobot.jussieu.fr

\section{Résumé}

Ce papier donne le détail d'un béquillage actif pour endoscope ou boroscope. Sa structure mécanique est tubulaire et basée sur un mécanisme hyper-redondant. Des actionneur en AMF avec leurs contrôleurs sont intégrés le long de cette structure pour produire les flexions voulues. En particulier, un comportement de type évitement d'obstacle est implémenté sur la base d'une détection des interactions entre l'instrument et son environnement.

Les actionneurs AMF sont deux ressorts plans en NiTi disposés en configuration antagoniste. Les mouvements articulaires sont produits des changements de phase martensite/austenite dans le matériau. Le comportement global de l'endoscope est contrôlé suivant une approche multi-agent.

\section{Introduction}

Un endoscope est un long appareil tubulaire pour l'inspection mini-invasive des cavités, canaux ou vaisseaux $[1,2]$. Il est inséré dans l'organisme par un orifice naturel ou pratiqué. Un diamètre extérieur courant est de $10 \mathrm{~mm}$ pour une longueur variant de 70 à $180 \mathrm{~mm}$. Le corps de l'endoscope contient plusieurs guides lumineux (typiquement deux), des canaux outil (pince pour biopsie, scalpel, outil de cytologie... ) et de l'optique et de l'électronique pour la retransmission d'images. Les endoscopes peuvent être rigides ou flexibles. Dans le cas de la video-endoscopie directe, l'image est transmise à l'opérateur via un paquets de fibres optiques. Dans le cas de la video-endoscopie indirecte, une caméra est montée à l'extrémité distale et l'image est visualisée sur un moniteur.

Une extrémité orientable peut être montée sur ces appareils. Elle permet de choisir un angle de vue particulier et peut aider à la progression de l'endoscope. Cette partie terminale est généralement actionnée par un ou deux câbles (en fonction du nombre de plans de flexion) reliés à un mécanisme déporté.

Des appareils similaires hors milieu médical, sont utilisés pour l'inspection visuelle de systèmes mécaniques hautement intégrés comme les moteurs d'avion ou les satellites. Dans ce cas, le boroscope doit pouvoir être inséré dans des passages étroits et les difficultés de progression rencontrées rejoignent celles des endoscopes.
Les instruments actuels d'endoscopie souffrent de limitations dues principalement aux manques de mobilités et de manoeuvrabilité dans les espaces géométriquement complexes ou exigüs. Ces instruments, bien que très flexibles, ne sont pas commandables. Il ne peuvent pas prendre de tournants sérés ni adopter des configurations 3D complexes. De ce fait, à l'heure actuelle, 60\% du circuit gastro-intestinal reste inatteignable avec les endoscopes de technologies classiques. De plus, un des risques majeurs avec ces instruments, dont la rigidité résiduelle n'est pas nulle, est la perforation des tissus. Enfin, il se pose le problème des contraintes mécaniques excessives appliquées aux câbles qui peuvent provoquer leur rupture ou leur déformation permanente.

Par ailleurs, la tendance actuelle est à la réduction continuelle des dimensions en vue d'accomplir des tâches d'inspection toujours plus délicates dans les domaines de la neuro-chirurgie, de la chirurgie cardio-vasculaire ou obstétricale. Ceci ne peut biensûr pas être entrepris par le biais d'une simple réduction d'échelle des technologies en places.

Ce papier décrit la conception d'un béquillage actif, de son système d'actionnement distribué et de la stratégie de commande associée pour l'inspection de cavités et de circuits 3D de géométrie complexe.

Très peu d'appareils de ce type sont évoqués dans la littérature [3,4,5]. Certains sont décrits dans le cadre de brevets [6,7]. Ils utilisent généralement les Alliages à Mémoire de Forme, des contrôleurs déposés sur des substrats flexibles et les techniques VLSI d'intégration.

\section{Description de la partie mécanique}

Le béquillage proposé est composé de maillons identiques (anneaux en acier) reliés les uns aux autres par des joints rotoïdes dont les axes sont alternés à $90^{\circ}$. Cette disposition permet de fléchir le corps de l'endoscope suivant n'importe quelle courbe 3D de l'espace pourvu qu'un rayon de courbure minimal soit respecté (environ 20mm). Les maillons sont obtenus par électro-érosion. 


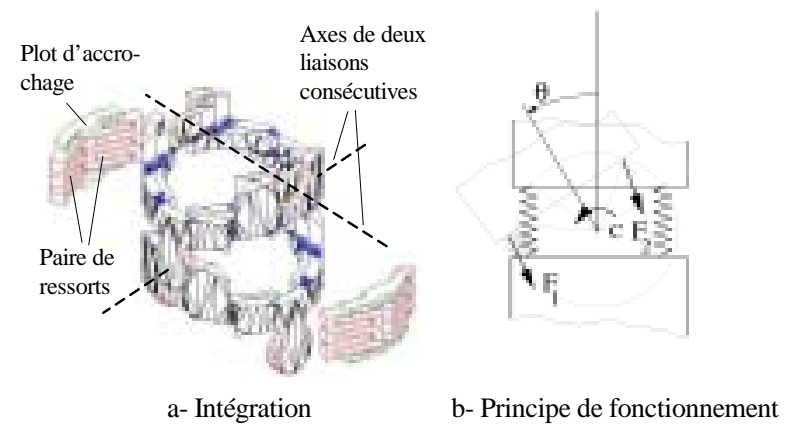

Figure 1. Description d'une articulation rotoïde

Par ce procédé, l'articulation et le corps du module ne forment qu'une seule pièce. L'arbre et l'alésage de la liaison sont respectivement un disque et son négatif, découpé dans un simple cylindre. Le blocage latéral est réalisé par l'insertion d'un anneau intérieur (Figure 1 a-). La distance d'un axe de liaison au suivant dans la chaîne est de $4 \mathrm{~mm}$.

L'actionneur d'une liaison est composé de deux paires de ressorts en AMF disposées de manière antagoniste par rapport à l'axe (Figure 1-b) et parfaitement intégrées à la structure. On commande une flexion en introduisant une différence entre les forces de rappel créées par les deux paires de ressorts. Pour cela, un courant électrique chauffe le matériau des ressorts situés du côté où l'on souhaite fléchir l'endoscope afin de produire un changement de phase et une rigidification de ce matériau. Le circuit de contrôle et d'alimentation est logé au niveau des plots d'accrochage des ressorts.

\section{Actionneurs en Alliage à mémoire de Forme}

Les ressorts formant les actionneurs sont des ressorts plans découpés par laser dans du ruban de $\mathrm{NiTi}_{50 \%-50 \%}$ (Figure 2-a).

Sur la Figure 2-b, on a modélisé un ressort comme un assemblage de lames (leaf) reliées les unes aux autres par des éléments rigides (link). Pour raison de symétrie, les parties rigides se translatent lors de l'étirement du ressort et les lames adoptent la double flexion que l'on a représentée sur la Figure 2-b.

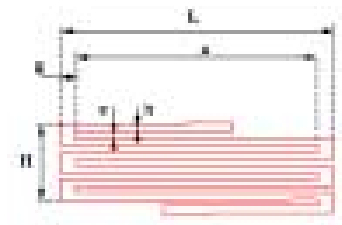

a- Géométrie

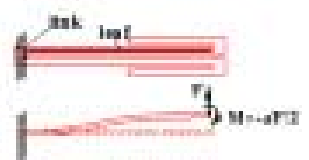

b- Modèle de déformation
Figure 2. Description des ressorts AMF

Basiquement, un changement de la rigidité de ces ressorts est obtenu en faisant passer leur matériau d'une phase dite martensite (souple) vers une phase dite austénite (impliquant une rigidité plus élevée) [8]. Ce changement de phase peut être atteint en élevant leur température par effet Joule. Les modules d'Young correspondant à chaque phase sont les pentes locales des deux courbes représentées sur la Figure 3. Ils valent environ $70 \mathrm{GPa}$ pour la phase austénite $\left(120^{\circ} \mathrm{C}\right)$ et vont de 1 à $20 \mathrm{GPa}$ pour la phase martensite $\left(20^{\circ} \mathrm{C}\right)$.

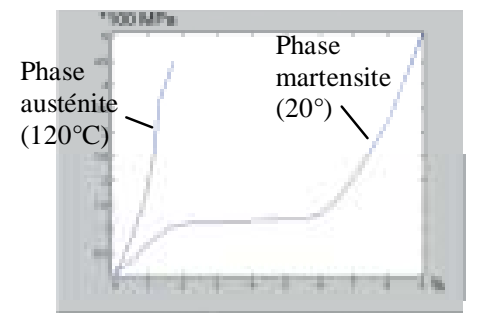

Figure 3. Diagramme contrainte-déformation du matériau

Pour le matériau employé ici $\left(\mathrm{NiTi}_{50 \%-50 \%}\right)$ et pour des contraintes nulles, les températures de transition sont voisines de $50^{\circ} \mathrm{C}$.

Les ressorts utilisés doivent être conçus de façon à produire un couple de sortie suffisant pour fléchir l'endoscope malgré la résistance de la gaine élastomère enveloppant le béquillage et celle des différents corps passant à l'intérieur de celui-ci (fibres optiques etc...). Cette résistance a été évaluée expérimentalement sur un endoscope existant (Figure 4).

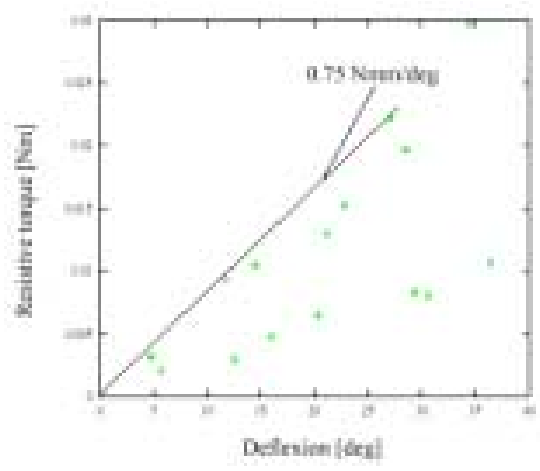

Figure 4. Résistance à la flexion d'une articulation d'un endoscope existant

$\mathrm{Au}$ niveau d'une articulation, un couple en sortie de $0,01 \mathrm{Nm}$ environ est nécessaire pour obtenir une flexion de $15^{\circ}$.

Sur la Figure 5, on a représenté la courbe du couple de sortie (torque) d'un actionneur complet (deux paires de ressorts antagonistes) ainsi que la contrainte maximale (stress) dans le matériau pour une flexion de $15^{\circ}$ de d'une articulation. Ces valeurs sont normalisées par rapport au couple de référence qui vaut $0,01 \mathrm{Nm}$ et une contrainte maximale admissible de $135 \mathrm{MPa}$ (fin du plateau martensite). En abscisse on fait varier la largeur L et la hauteur $\mathrm{H}$ des ressorts (Voir Figure 2-a).

Le meilleur compromis revient à choisir des valeurs de $\mathrm{L}=2,35 \mathrm{~mm}$ et $\mathrm{H}=2,00 \mathrm{~mm}$ qui correspondent à un couple de sortie supérieur à $0,008 \mathrm{Nm}$ et à une contrainte maximale inférieure à $145 \mathrm{MPa}$. Les autres dimensions des ressorts se déduisent de celles-ci en tenant compte des contraintes de réalisation par découpe laser et valent : $\mathrm{g}=0,135 \mathrm{~mm}$, $\mathrm{e}=0,25 \mathrm{~mm}$ et $\mathrm{h}=0,125 \mathrm{~mm}$. 


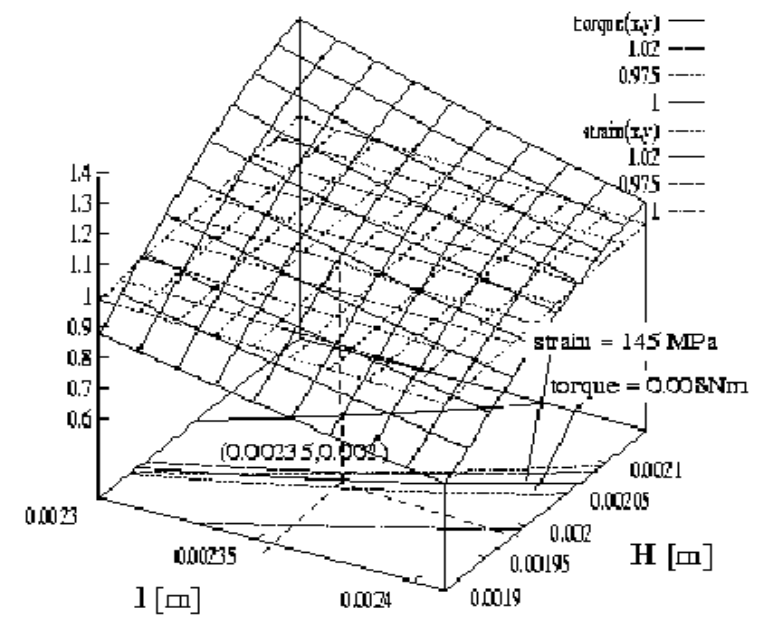

Figure 5. Calcul des dimensions extérieures des ressorts.

\section{Choix d'un schéma de commande}

\subsection{Commande en puissance}

$\mathrm{Au}$ plus bas niveau, on commande la puissance injectée dans les ressorts lorsqu'un mouvement du béquillage est requis. Cette injection peut être réalisée par une commande en tension en boucle ouverte si l'on connaît la résistance électrique des ressorts. Cependant, une variation de $20 \%$ de cette résistance en fonction de la température du matériau est courante [8,9]. Il est donc préférable d'introduire une estimation en-ligne de cette résistance comme illustré sur la Figure 6.

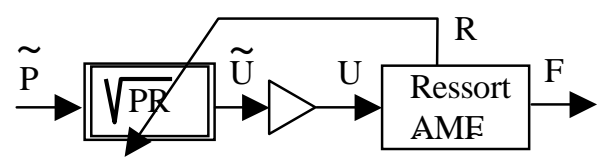

Figure 6. Commande en puissance d'un ressort AMF

\subsection{Aspects énergétiques}

Dans un actionneur tel que celui décrit précédemment, quand les deux paires de ressorts antagonistes sont activées de la même manière et en même temps, cela ne produit aucun mouvement de la structure. Par contre, des efforts internes importants peuvent apparaître. Ce cas de figure est à proscrire du point de vue énergétique et peut, de plus, endommager la structure.

A fin de limiter la génération des efforts internes, il faut éviter l'activation simultanée des ressorts $\mathrm{AMF}$ antagonistes. Cela signifie que l'on ne doit pas injecter de la puissance électrique dans une paire de ressorts tant que la paire de ressorts antagonistes n'a pas été suffisamment refroidie et que son matériau n'a pas retrouvé un état proche de l'état martensite (état de souplesse relative).

La connaissance en-ligne de l'état du matériau AMF constituant les ressorts est nécessaire pour appliquer ce principe. Une première possibilité est de mettre en place une mesure instantanée de leur température. Celle-ci est cependant difficile en pratique (constantes de temps importantes, problèmes d'intégration des thermocouples ou thermistances). La solution que nous proposons est basée sur une lecture en-ligne de la résistance électrique que présente le matériau.

Sur la Figure 7 nous avons reporté la résistance électrique $\mathrm{R}$ d'un fil en $\mathrm{AMF}$ passant de l'état martensite à l'état austénite par chauffage puis de l'état austénite à l'état martensite par refroidissement. Deux seuils $\left(\mathrm{R}_{\mathrm{M}_{\rightarrow}} \mathrm{A}\right.$ and $\mathrm{R}_{\mathrm{A}_{\rightarrow} \mathrm{M}}$ ) matérialisent la fin de ces transitions.

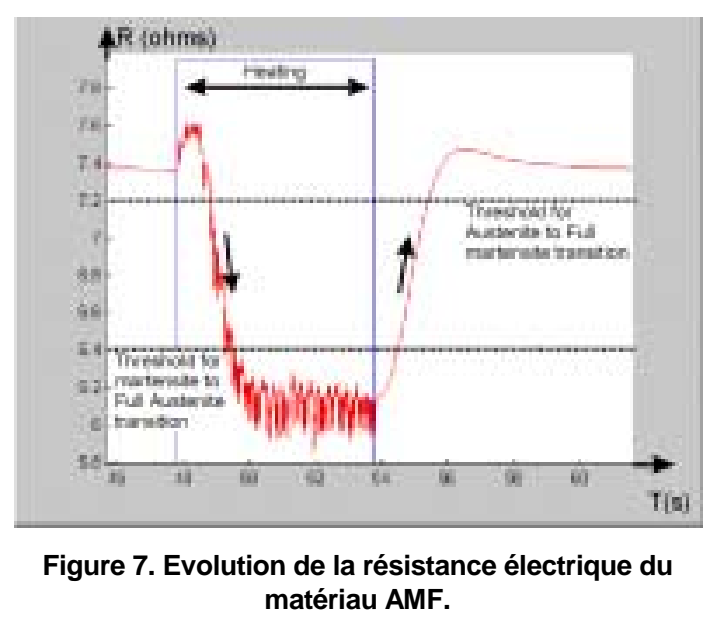

Comme on le voit, la connaissance de la résistance électrique du matériau est un bon indicateur de son état. Sur cette base, il est possible d'inhiber l'injection de puissance dans un actionneur AMF lorsque celle-ci risque de produire une élévation importante des efforts internes. C'est le rôle de l'élément de couplage ajouté au schéma de contrôle basniveau proposé précédemment (Figure 8).

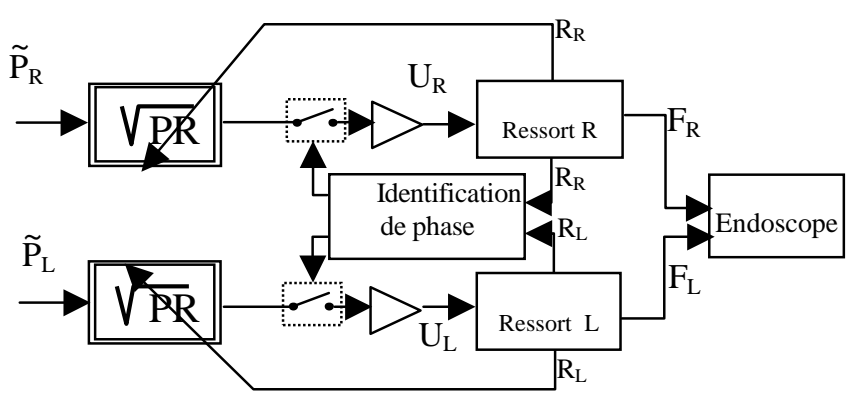

Figure 8. Couplage du contrôle bas niveau de deux matériaux AMF en configuration antagoniste.

\subsection{Contrôle de l'inclinaison}

La commande en position d'un actionneur comme celui que nous proposons se fait en boucle fermée sur la base d'une mesure de la position (inclinaison) et d'un contrôleur de niveau articulaire.

Il existe dans la littérature de nombreuses solutions pour le contrôle en position des actionneurs en AMF. Les plus avancées de ces solutions prennent en compte une modélisation physique du matériau $\mathrm{AMF}$ considéré qui 
inclut les dérives, l'hystéresis et autres non-linéarités $[10,11,12]$. De telles approches sont très coûteuses en terme de temps d'identification et de réglage. Elles sont donc inadaptées dans le cas présent qui est de contrôler la courbure d'un endoscope doté d'un nombre important d'actionneur.

Nous nous sommes intéressés à des solutions plus simples et plus générales. En particulier celles représentées sur la Figure 9. Il s'agit d'un simple contrôleur linéaire PI et d'un contrôleur à structure variable basique de type tout ou rien $(\mathrm{ON} / \mathrm{OFF})$.

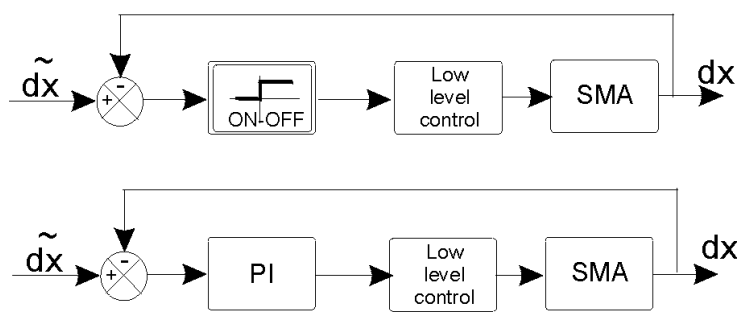

Figure 9. Deux contrôleurs élémentaires pour la commande en position d'actionneurs en AMF.

Les performances de ces deux contrôleurs sont comparés sur la Figure 10. Nous les avons appliqués à la commande en position d'un simple fil de NiTi dont on cherche à contrôler la contraction.

Les temps de monté (contraction par échauffement) sont de $0,4 \mathrm{~s}$ environ dans les deux cas avec un léger dépassement pour le contrôleur tout ou rien. Les erreurs statiques sont presque nulles à chaque fois. Les temps de descente (passage de la phase austénite à la phase martensite par refroidissement) sont beaucoup plus grands que les temps de monté (environ 2,2s). Dans les deux cas, il s'agit d'un refroidissement par convexion naturelle. Un refroidissement par convexion forcée réduirait significativement ces temps de descente comme dans [5] ou [9].

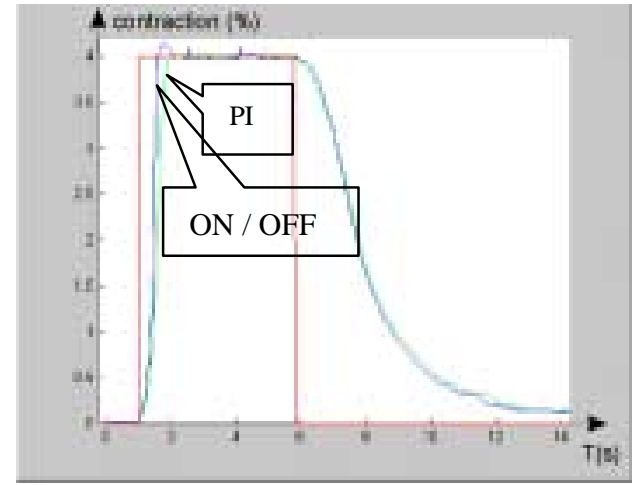

Figure 10. Comparaison des performances des deux contrôleurs élémentaires.

Comme on peut le voir, le contrôleur PI produit globalement une trajectoire plus lisse que le contrôleur ON/OFF. Cependant, les Alliages à Mémoire de Forme sont des matériaux actifs dont le comportement est fondamentalement non-linéaire. En conséquence, le réglage de gain du contrôleur PI proposé est fortement dépendant de la configuration. A l'opposé, le contrôleur ON/OFF ne nécessite pas de réglage de gain.

\section{Validation expérimentale du schéma de commande}

En conséquence de tout ce qui précède, la solution retenue pour la commande en position de nos actionneurs AMF comprendra :

1. un asservissement bas niveau en puissance basé sur une estimation en ligne de la résistance électrique (Figure $6)$.

2. une "commande gardée" pour limiter la production d'efforts internes à la structure (Figure 8).

3. un asservissement en position utilisant un contrôleur élémentaire de type $\mathrm{ON} / \mathrm{OFF}$.

Les actionneurs à base de ressorts AMF étant encore en phase de réalisation, la solution proposée a été testée sur un banc d'essai comprenant la structure béquillée du chapitre 2 actionnée par quatre fils de NiTi antagonistes deux à deux.

La Figure 11 montre la réponse de cet endoscope pour une flexion commandée dans le plan horizontal. Sur le graphe de la réponse temporelle, on constate que les temps de parcours sont de l'ordre de la seconde ce qui est acceptable vu le type d'application visé. On note en particulier un temps de $1,5 \mathrm{~s}$ pour effectuer le basculement complet de la structure entre la position $80^{\circ}$ et $-80^{\circ}$.

Par ailleurs, on remarquera l'influence limité de l'application de la commande gardée sur le comportement général de l'asservissement en position. Celui-ci se matérialise par des fenêtres de temps durant lesquelles l'activation de tel ou tel fil AMF est inhibée du fait de l'état austénitique de son vis à vis.

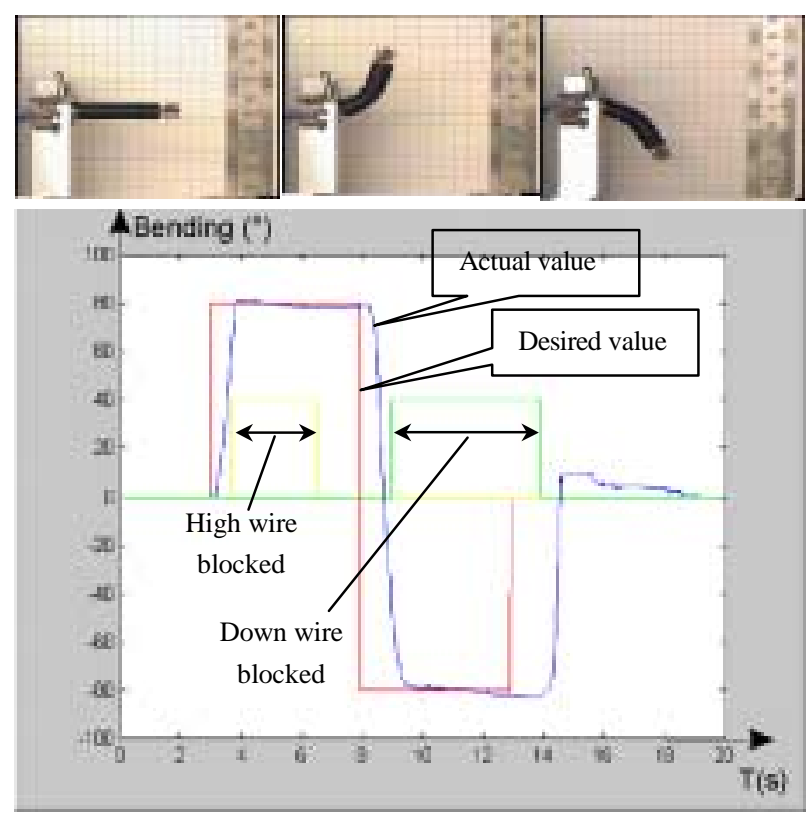

Figure 11. Résultats de la commande en flexion d'un endoscope "4 fils". 


\section{Planification de trajectoire}

Un endoscope basé sur une structure poly-articulée telle que celle décrite au chapitre 2 est destinée à progresser dans des environnements éxigüs tel le milieu intestinal. Il est nécessaire d'y parvenir tout en minimisant les interactions physiques avec cet environnement de manière à limiter les risques de perforation.

Il faut donc parvenir à contrôler en temps réel la courbure de l'endoscope sur la base d'une détection des obstacles en contact ou proches d'entrer en contact avec lui.

Une résolution algébrique de ce problème est extrêmement complexe dans le cas d'une structure hyperredondante et à fortiori, dans le cas d'un environnement peu connu. Une solution de type comportement réactif est donc préférable ici.

La solution que nous proposons se base sur un découpage virtuel de l'endoscope en un ensemble de sous systèmes indépendants considérés comme des agents [13]. Chaque agent est capable de détecter un contact avec l'environnement et de modifier de manière adaptée la courbure de l'endoscope localement.

Cette approche simple et modulaire est indépendante de la longueur de l'endoscope. De plus, il s'agit d'une stratégie distribuée qui minimise la quantité d'informations échangées entre les étages de la structure. Trois types différents de comportements locaux sont décrits sur la Figure 12. Ils correspondent à des sous-systèmes composés de 1, 2 ou 3 maillons du béquillage.
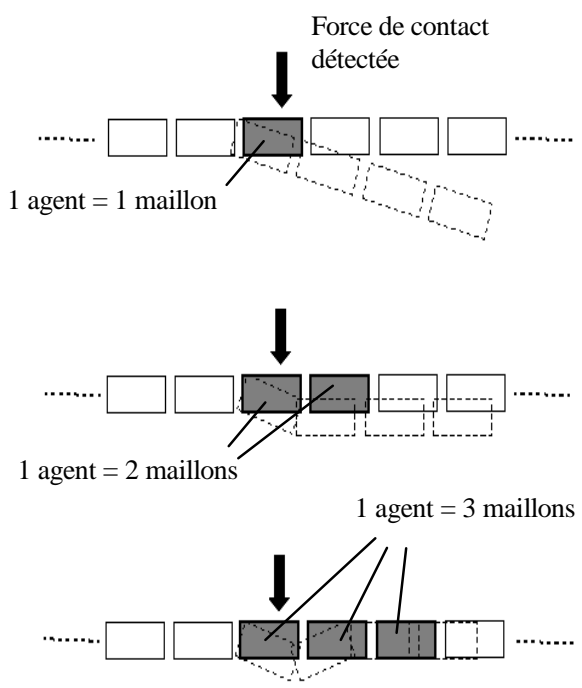

Figure 12. Trois stratégies de réaction aux forces de contact.

Dans le cas 1 agent = 1 maillon, l'actionneur du maillon qui perçoit le contact est sollicité pour produire une inclinaison locale de la structure dans le sens voulu. Dans le cas 1 agent $=2$ maillons ou 1 agent $=3$ maillons, alors le ou les actionneurs supplémentaires sont également sollicités pour améliorer le mouvement produit dans le sens d'une moindre perturbation de la configuration globale de l'endoscope.

Le comportement d'une structure planaire (béquillage composé de vingt maillons) progressant dans un conduit et guidée uniquement par l'action locale des agents a été testé en simulation. Dans chaque cas, une force de contact détectée au niveau d'un maillon est interprétée comme la présence d'un obstacle. Il est à noté que dans le cas d'un agent constitué de plusieurs maillons, il est nécessaire de disposer d'un canal de communication entre les maillons pour la transmission des informations.

Comme on le voit sur la Figure 13, l'approche purement locale ( 1 agent $=1$ maillon) conduit à l'instabilité. La seconde et la troisième solutions ( 1 agent $=2$ ou 3 maillons) préservent la stabilité de l'endoscope. On remarque par ailleurs, que plus l'agent comprend un nombre important de maillons, plus la distance parcourue dans le conduit est grande.
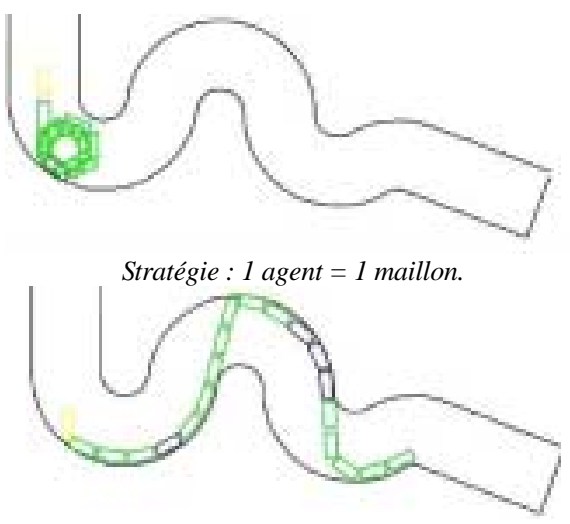

Stratégie $: 1$ agent = 2 maillons.

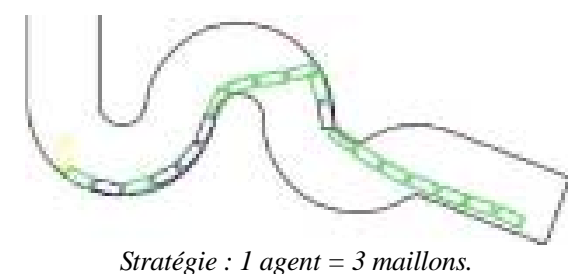

Figure 13. Simulations d'une progression avec évitement d'obstacle.

\section{Conclusions et perspectives}

A ce stade de notre travail, le prototype d'endoscope multi-degrés de liberté et autonome que nous souhaitons réaliser est entièrement défini en termes de structure mécanique, d'actionneur et contrôle bas niveau.

La partie capteur de contact est en cours de développement. Un dimensionnement du transducteur est actuellement à l'étude au LRP6 sur la base de différentes technologies : piezzo-résistive, piezzo-électrique, polymère dopé.

Le développement de la partie comportement intelligent est en voie d'achèvement. A ce titre, des simulations 3D en 
milieu réaliste sont menées. Elles intègrent l'ensemble des données disponibles sur le prototype envisager : mécanique, comportement des actionneurs, interaction endoscope/milieu, interaction opérateur/endoscope etc...

Un premier prototype opérationnel complet d'endoscope devrait voir le jour prochainement.

\section{Références}

[1] R. Azziz, A. Alvarez, A., "Operative laparoscopy and hysteroscopy 2nd edition ", Springer-Verlag, NewYork, 1997.

[2] D. Dunn, Ch. Watson, "Laparoscopic Cholecys-tectomy Problems and Solutions", Blackwell Scientific Publications, Oxford, 1992.

[3] D. Reynaerts, J. Peirs, H. Van Brussel, "Design of a Shape Memory Actuated Gastrointestinal Intervention System", 6th International Symposium on Micro Machine and Human Science, 1995.

[4] P. Dario, C. Paggetti, N. Troisfontaine, E. Papa, T. Ciucci, M. Carrozza, M. Marcacci, "A Miniature Steerable End-Effector for Application in an Integrated System for Computer-Assisted Arthroscopy", International Conference on Robotics and Automation IEEE, 1573-1579, 1997.

[5] Y. Nakamura, A. Matsui, T. Saito, K. Yoshimoto, "Shape Memory Alloy Active Forceps for Laparoscopic Surgery", International Conference on Robotics and Automation IEEE, 2320-2327, 1995.

[6] S. Takayama, "Method of Manufacturing a MultiDegree-of-Freedom Manipulator", US Patent No 5,679,216, 1997.

[7] R. Maynard, "Spatially Distributed SMA Actuator Film providing unrestricted Movement in Three Dimensional Space"., US patent No 5,405,337, 1995.

[8] J. Hesselbach, H. Stork, "Electricly Controled Shape Memory Actuators for Use in Handling Systems", International Conference on Shape Memory and Superelasticity Engineering and Biomedical Applications, 1994.

[9] K. Ikuta, M. Tsukamoto, S. Hirose, "Shape Memory Alloy Servo Actuator System with Electric Resistance Feedback", International Conference on Robotics and Automation IEEE, 427-430, 1988.

[10]A. Krupa, G. Morel, M. Mathelin, "Modeling and Vision Based Control of a Micro Endoscope Head Actuated with Shape Memory Alloy Wires", International Advanced Robotics Programme Workshop on Micro Robots, Micro-machines and Systems, 122-127, 1999.

[11] H. Benzaoui, C. Lexcellent, N. Chaillet, B. Lang, A. Bourjault, L. Thiery, "Experimental and Theoritical Study of Shape Memory Alloy Wire Actuator" Journal of intelligent material systems and structures 8, 7: 619629, 1997.

[12] N. Troisfontaine, Ph. Bidaud, "Position and Force Control of SMA Micro-Actuators", International Advanced Robotics Programme - Second International Workshop on Micro-Robotics and Systems, 1998.
[13] D. Duhaut, "Using a Multi-agent Approach to Solve the Inverse Kinematics", Intelligent Robot and System Conference, IROS, 1993. 